\title{
Mixed-Size Diamond Seeding for Low-Thermal-Barrier Growth of CVD Diamond onto GaN and AlN
}

\author{
E. J. W. Smith ${ }^{1}$, A. H. Piracha ${ }^{1,6}$, D. Field ${ }^{2}$, J. W. Pomeroy ${ }^{2}$, G. R. Mackenzie ${ }^{1,3}$, \\ Z. Abdallah ${ }^{2}$, F. C-P. Massabuau ${ }^{4,5}$, A. M. Hinz ${ }^{4}$, D. J. Wallis ${ }^{4,7}$, R. A. Oliver ${ }^{4}$, M. Kuball ${ }^{2}$, \\ P. W. May ${ }^{1, *}$.
}

${ }^{1}$ School of Chemistry, University of Bristol, Bristol BS8 1TS, United Kingdom.

${ }^{2}$ Center for Device Thermography and Reliability, H.H. Wills Physics Laboratory, University of Bristol, BS8 1TL, United Kingdom.

${ }^{3}$ Interface Analysis Centre, HH Wills Physics Laboratory, University of Bristol, BS8 1TL, United Kingdom.

${ }^{4}$ Department of Materials Science and Metallurgy, University of Cambridge, Cambridge CB3 0FS, United Kingdom

${ }^{5}$ Department of Physics, SUPA, University of Strathclyde, Glasgow G4 0NG, United Kingdom

${ }^{6}$ John A. Paulson School of Engineering and Applied Sciences, Harvard University, Cambridge, MA 01238, USA.

${ }^{7}$ Centre for High Frequency Engineering, University of Cardiff, The Queens Building, Cardiff, CF24 3AA, United Kingdom

* Email: Paul.May@bristol.ac.uk

\begin{abstract}
We report a method of growing a diamond layer via chemical vapour deposition (CVD) utilizing a mixture of microdiamond and nanodiamond seeding to give a low effective thermal boundary resistance (TBR eff) for heat-spreading applications in high-frequency, high-power electronic devices. CVD diamond was deposited onto thin layers of both GaN and AlN on Si substrates, comparing conventional nanodiamond seeding with a two-step process involving sequential seeding with microdiamond then nanodiamond. Thermal properties were determined using transient thermoreflectance (TTR), and the samples were also analysed with SEM and X-ray tomography. While diamond growth directly onto GaN proved to be unsuccessful due to poor adhesion, films grown on AlN were adherent and robust. The twostep mixed-seeding method gave TBR eff values $<6 \mathrm{~m}^{2} \mathrm{~K} \mathrm{GW}^{-1}$ that were 30 times smaller than for films grown under identical conditions but using nanodiamond seeding alone. Such
\end{abstract}


remarkably low thermal barriers obtained with the mixed-seeding process offer a promising route for fabrication of high-power GaN HEMTs using diamond as a heat spreader with an AlN interlayer.

\section{Introduction}

Gallium nitride $(\mathrm{GaN})$ electronic devices are in high demand for high-power, high-frequency applications due to their outstanding properties [1,2]. For instance, GaN high electron mobility transistors (HEMTs) have recently drawn attention for their use in, among other industries, transmitter base-stations for communications [3]. However, poor thermal management of these devices is preventing them from realising their full potential. Significant, localised, Joule heating in the channel of the devices results in degradation of device performance and a huge decrease in their reliability [4,5]. For GaN HEMTs to play a part in the drive for $5 \mathrm{G}$ communications and beyond, improved thermal management is a necessity for the devices to operate at the required power densities with an acceptable lifetime. One solution to this is to attach the device to a high thermal conductivity material which can rapidly transfer the localised heat to an external cooling system or radiator.

To clarify terminology, interfacial thermal resistance is defined as the temperature difference between two materials divided by the heat flow across the interface area - with units of $\mathrm{m}^{2} \mathrm{~K} \mathrm{~W}^{-1}$; thermal conductivity is heat flow rate across a thickness of material divided by the temperature difference - with units of $\mathrm{W} \mathrm{m}^{-1} \mathrm{~K}^{-1}$. The interfacial thermal resistance depends on the acoustic similarity of the two materials as well as the quality of the interface, whilst the thermal conductivity is an intrinsic material property.

Commercial GaN devices currently use a technology based on thin films ( $2 \mu \mathrm{m}$ thick) of $\mathrm{GaN}$ deposited onto a silicon carbide (SiC) wafer with a thin nucleation layer [6,7]. Although the thermal conductivity values for bulk GaN $\left(160 \mathrm{~W} \mathrm{~m}^{-1} \mathrm{~K}^{-1}\right)$ and $\mathrm{SiC}\left(\sim 420-490 \mathrm{~W} \mathrm{~m}^{-1} \mathrm{~K}^{-1}\right)$ are reasonably high, the nucleation layer and the resulting microstructure near to the interface can cause a significant barrier for heat to travel from the $\mathrm{GaN}$ device to the $\mathrm{SiC}$ substrate [8]. To quantify this, the parameter $\mathrm{TBR}_{\mathrm{eff}}$ defines the effective thermal boundary resistance, which is a combined thermal resistance term, typically including the intrinsic thermal boundary resistance at each interface, the resistance of any thin-film adhesion layer, plus any other factors (such as a poor-quality region near the interface) that may affect thermal transport across the layer(s) [8]. 
Ultimately, the heat must be removed via the back or sides of the substrate wafer to an external cooling system. Replacing the conventional $\mathrm{SiC}$ substrates with a high-conductivity substrate, such as diamond ( $2000 \mathrm{~W} \mathrm{~m}^{-1} \mathrm{~K}^{-1}$ [7]), allows for a 3 times increase in RF output power density [9] and greatly improved device performance and lifetime [7,10].

Ideally, to reduce $\mathrm{TBR}_{\text {eff, }}$, the $\mathrm{GaN}$ device would simply be deposited directly onto a diamond substrate, thus eliminating any thermal resistance from a barrier layer. However, large-area high-quality diamond substrates are not yet widely available. Even if they were, depositing device-quality $\mathrm{GaN}$ onto diamond is difficult [11], mainly because $\mathrm{GaN}$ has a hexagonal wurtzite structure while diamond has a cubic diamond structure. A Japanese group reported single-crystal AIN growth on single-crystal (111) diamond substrates using metalorganic vapour-phase epitaxy [12,13]. They subsequently used this single-crystal AIN as a buffer layer to deposit epitaxially AlGaN/GaN HEMTs on diamond, and successfully demonstrated high-power RF operation at $1 \mathrm{GHz}$ with a significant reduction in deviceoperation temperature $[14,15,16,17]$. Similarly, a European group deposited epitaxial AlGaN/GaN HEMT structures on (111) diamond using molecular beam epitaxy [18,19]. The HEMTs exhibited outstanding RF performance anda cut-off frequency of $21 \mathrm{GHz}$. Nevertheless, without expensive and sophisticated deposition systems such as those mentioned above, growth of $\mathrm{GaN}$ onto diamond remains problematic and often results in poor device performance [20].

Conversely, depositing diamond onto $\mathrm{GaN}$ is also tricky for a number of reasons. First, diamond CVD utilises high temperatures (typically $800^{\circ} \mathrm{C}$ and above) and a gaseous environment rich in atomic hydrogen [21]. Under these conditions, atomic hydrogen can etch $\mathrm{GaN}$ (to form $\mathrm{NH}_{3}$ and liquid $\mathrm{Ga}$ ), preventing diamond deposition. This etching can be prevented if the GaN surface is seeded with nanodiamond (ND) sufficiently densely that the lateral diamond growth rate exceeds the etch rate. In this case, a protective diamond layer grows to cover the GaN surface before significant etching can occur. Even with these measures, the interface between $\mathrm{GaN}$ and diamond is rather weak because Ga does not form a carbide [22]. Thus, the diamond adheres to the GaN mainly via weak van der Waals interactions, rather than strong covalent bonds, making it prone to delamination. This becomes a serious problem when cooling the samples down from the diamond CVD temperatures, because GaN has a roomtemperature thermal expansion coefficient $\left(5.59 \times 10^{-6} \mathrm{~K}^{-1}\right)$ that is $28 \%$ larger than that of diamond $\left(4.38 \times 10^{-6} \mathrm{~K}^{-1}\right)$ [23]. Upon cooling, the $\mathrm{GaN}$ will contract back to its roomtemperature volume while the diamond to which it is bonded contracts far less. This causes 
compressive stresses to be developed in the diamond layer, leading either to unwanted bowing of the sample or to delamination of the entire diamond layer [21].

To overcome some of these growth and adhesion problems, thin buffer layers of $\mathrm{SiC}$ or $\mathrm{SiN}$ have been used between the diamond and $\mathrm{GaN}$ [10]. However, these layers often have poor thermal conductivity, and so create a thermal bottleneck [7]. A workable compromise is to use AlN as the barrier layer. Recent work [24] showed that diamond growth onto AlN is a possible pathway forward; thick $(100 \mu \mathrm{m})$ diamond films deposited using a standard NDseeding approach were reported with low TBR eff values of $<6 \mathrm{~m}^{2} \mathrm{~K} \mathrm{GW}^{-1}$. As such, AlN-onSi substrates were chosen for nucleation studies because AlN is compatible with the diamond CVD conditions and readily forms a carbide which helps diamond adhesion [21], plus bulk AlN benefits from having higher thermal conductivity than $\mathrm{SiN}\left(\sim 250 \mathrm{~W} \mathrm{~m}^{-1} \mathrm{~K}^{-1}[25]\right.$ and $\sim 30 \mathrm{~W} \mathrm{~m}^{-1} \mathrm{~K}^{-1}$, respectively). An added benefit is that AlN can act as an etch-stop layer and an adhesion layer for the diamond growth [26].

A further issue with using diamond as a heat spreader for $\mathrm{GaN}$ devices is the potentially high TBR of the diamond seeding layer. The dense ND seeding layer results in the first $\sim 0.5 \mu \mathrm{m}$ of the diamond film being composed of nm-sized crystallites with a high grain-boundary density and very poor thermal conductivity [27]. This is mitigated somewhat as the diamond film grows thicker, because the crystallite sizes and thus, the thermal conductivity, both increase to approach those of bulk diamond. But even with thick diamond films, the initial $0.5 \mu \mathrm{m}$ nucleation layer still has a very high intrinsic $\mathrm{TBR}_{\mathrm{eff}}$, which reduces the advantages of using diamond as a heat spreader [28].

In this paper, we report the results of a new seeding approach for deposition of diamond onto $\mathrm{GaN}$ (and other materials), which provides both the necessary protective layer to prevent the $\mathrm{GaN}$ from etching while significantly lowering the overall $\mathrm{TBR}_{\mathrm{eff}}$. We employ a mixed seeding method which comprises a two-step process (see Figure 1). First, the sample is seeded with microdiamond (MD) particles. Because these MD particles have smooth facets, they have a large contact area with the underlying surface, ideal for good thermal transport across this interface. The presence of these large particles also partially eliminates the detrimental effects

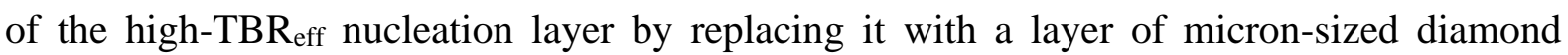
crystallites with higher conductivity. However, the downside of using larger seeds is that the gaps between them are also larger, allowing $\mathrm{H}$ atoms to etch any exposed $\mathrm{GaN}$ surface during CVD. Hence, the second step is to seed again, this time with ND, which fills in the gaps between the larger seeds, removing voids at the interface and protecting the surface. 
Here, we have investigated the effectiveness of this approach, using CVD diamond deposited onto simple GaN-on-silicon and onto AlN-on-silicon wafers. This allows the diamond-to$(\mathrm{Ga} / \mathrm{Al}) \mathrm{N}$ interface properties to be studied without the further complexities of full device structures.

\section{(a)}
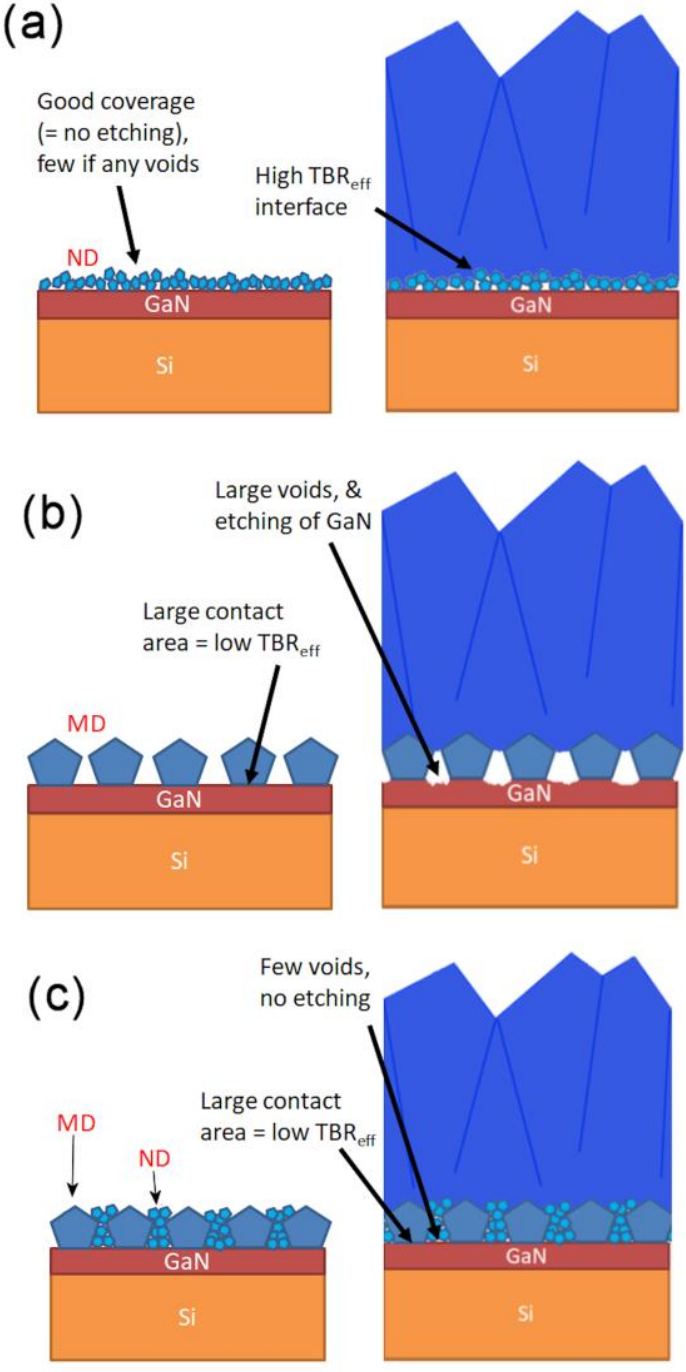

Figure 1. Schematic diagram explaining the rationale for two-step seeding. The thin AlGaN strain-control buffer layer between the $\mathrm{GaN}$ and $\mathrm{Si}$ has been omitted for clarity. The left-hand panels show the seeding method, while the right-hand panels are after subsequent diamond CVD. (a) Seeding with ND alone provides good surface coverage with few, if any, surface areas exposed. Upon diamond growth, there is little or no etching of the GaN surface, but the nucleation layer of ND has a high $\mathrm{TBR}_{\text {eff. }}$ (b) Conversely, seeding with only MD provides large-surface-area contacts and therefore efficient heat transfer, but the exposed GaN surface creates voids at the interface as well as suffering etch damage during diamond CVD. The voids reduce the adhesion at the interface making the film more prone to delamination, as 
well as increasing the $\mathrm{TBR}_{\text {eff }}$ hugely. (c) Two-step seeding (MD followed by ND) allows the benefits of both - a large contact area and therefore low TBR eff, plus very few voids and no etching.

\section{Method}

Epitaxial layers of either GaN or AlN were deposited onto single-crystal Si (111) handle wafers (1 mm thick) using metal-organic chemical vapour deposition (MOCVD). For the GaN samples the layers consisted of $\sim 1 \mu \mathrm{m}$ of $\mathrm{GaN}$ on a $1.7 \mu \mathrm{m}$-thick graded $\mathrm{AlGaN}$ strain-control buffer layer, while for the AlN samples the layer structure was simply $130 \mathrm{~nm}$ AlN directly grown onto the Si substrate. In both cases, the single-crystalline epitaxial layer was (0001)oriented with a surface RMS roughness of $<1 \mathrm{~nm}$ over $5 \times 5 \mu \mathrm{m}$ scans.

The wafers were cut into $10 \times 10 \mathrm{~mm}$ squares and then pre-seeded with diamond using an electrostatic spray technique [29]. This technique utilises a suspension of diamond particles in methanol, which is sprayed onto the grounded substrate using an electrostatic potential difference of $\sim 35 \mathrm{kV}$. When optimised, this results in a near monolayer coating of close-packed seeds uniformly covering the substrate surface. The seeding was performed using two types of diamond particles: the first was $2.0 \pm 1.0 \mu \mathrm{m}$ microdiamond (DIADUST, PM 1-3, van Moppes, Switzerland), which were offcuts from polishing and cutting natural diamond gemstones, while the second was detonation nanodiamond (Amando, Japan [30]) with size distribution $3.3 \pm 0.6 \mathrm{~nm}$ ) supplied as $1 \mathrm{wt} \%$ suspension in water.

Seeding was first optimised on $\mathrm{Si}$ substrates to produce close-packed monolayers of both diamond particle types, in turn, by varying the electrospray deposition conditions (voltage, nozzle diameter, nozzle-substrate distance, etc.). For MD seeding, a variable mass $(m=5$ $10 \mathrm{mg}$ ) of the MD powder was dispersed in $1 \mathrm{~cm}^{3}$ of water. This suspension was added to $25 \mathrm{~cm}^{3}$ of methanol and then sonicated using an ultrasonic probe for $2 \mathrm{~h}$ to form a suspension and to deaggregate any clusters. $1 \mathrm{~cm}^{3}$ of this suspension was then electrosprayed onto the substrate sequentially $x_{\mathrm{MD}}=1$ to 3 times. For ND seeding, A variable number of drops $(n=10$ 50 ) of the ND suspension was added to $20 \mathrm{~cm}^{3}$ of methanol, and sonicated as above. $1 \mathrm{~cm}^{3}$ of this suspension was then electrosprayed sequentially onto the MD-seeded substrates $x_{\mathrm{ND}}=1$ to 5 times. 
We use the term 'MD+ND' to describe the new two-step process comprising seeding sequentially with MD then ND. The concentrations of ND $(n)$ and MD $(m)$ suspensions, and the number of spray doses, $x_{\mathrm{ND}}, x_{\mathrm{MD}}$, respectively, were all varied by trial-and-error to optimise the nucleation process. The aim was to obtain a close-packed near-monolayer of MD first, and then to fill in any voids with ND. For evaluation of the results, comparative images of the seeded surfaces (pre-growth) would have been preferred, but the 1000-fold difference in size between the MD and ND seeds made it problematic to resolve both seed-types simultaneously in an SEM. Atomic force microscopy also proved problematic for the same reasons, and also because the probe tip tended to push the seeds around the surface rather than profile them. Instead, it was simpler to evaluate the seeding visually using SEM images of diamond films grown following CVD on the differently seeded Si substrates. Diamond films $>10 \mu \mathrm{m}$ thick grown from these seeds became difficult to distinguish from one another visually; they all looked like high-quality, large-grained faceted microcrystalline diamond films with no signs of the underlying seeding. Therefore, shorter CVD growth runs producing films $\sim 3-4 \mu \mathrm{m}$-thick were used for the evaluation of the seeding as the initial stages of growth could be clearly seen.

Diamond growth was performed using microwave plasma-enhanced CVD (MWCVD) in a $2.45 \mathrm{GHz}, 1.5 \mathrm{~kW}$ ASTeX-type reactor. Growth occurred in a methane/hydrogen plasma (4\% $\mathrm{CH}_{4}$ in $\mathrm{H}_{2}$ ) with MW input power $1100-1500 \mathrm{~W}$, chamber pressure 110-150 Torr, yielding substrate temperatures $750-950^{\circ} \mathrm{C}$ (determined using calibrated one-colour pyrometry). Growth duration varied depending on the thickness of diamond required for the specific experiment, ranging from $1-50 \mathrm{~h}$, and with a growth rate $\sim 0.5 \mu \mathrm{m} \mathrm{h}^{-1}$ at $750^{\circ} \mathrm{C}$ and $\sim 2 \mu \mathrm{m} \mathrm{h}^{-1}$ at $950^{\circ} \mathrm{C}$. Laser Raman analysis of all the as-grown films was performed using a Renishaw 2000 spectrometer with $514 \mathrm{~nm}$ laser excitation.

After growth, samples for transient thermoreflectance (TTR) analysis were coated with a $10 \mathrm{~nm}$ $\mathrm{Cr}$ adhesion layer followed by a $100 \mathrm{~nm}$-thick Au transducer layer for measurement of TBR $\mathrm{eff}_{\mathrm{f}}$ via TTR using the equipment and method detailed previously [3,31]. In this setup, a $355 \mathrm{~nm}$ pulsed-laser (10 ns pulse duration, $30 \mathrm{kHz}$ repetition rate, $\sim 50 \mu \mathrm{m}$ spot diameter) was used to periodically heat the material surface, whilst a continuous-wave $532 \mathrm{~nm}$ probe laser $(\sim 2 \mu \mathrm{m}$ spot diameter) tracked the resulting reflectivity change. This is linearly proportional to the temperature of the material surface. Fitting a model to the measured data allows the thermal material parameters of the film to be determined [32]. One limitation of this method is that it relies on transmission of laser light through the film, and so if the surface is too rough the light scatters and no measurements can be made. For polycrystalline diamond film analysis, 
therefore, thin films were preferred as these had smoother surfaces. To assess run-to-run and across-sample uniformity, three samples were grown for each variant of deposition conditions, and then TTR was performed in three places on each sample. After TTR analysis, the films were laser cut so that cross-sectional SEM could be performed to determine the thickness of the diamond layer.

$\mathrm{X}$-ray tomography (XRT) was used to take 4500 sequential X-ray slices over a $360^{\circ}$ rotation of the sample at equal intervals of $\sim 0.08^{\circ}$, without the need for mechanical sectioning. In this way, 3D representations of the sample were compiled allowing the densities of the different layers of the structure to be distinguished. The XRT system used was a Zeiss XRadia Versa 520 , operated at $80 \mathrm{kV}$ and $7 \mathrm{~W}$ with a $40 \times$ objective lens, giving a voxel resolution of $\sim 1.5 \mu \mathrm{m} \times 1.5 \mu \mathrm{m}$ in the plane of the surface and a depth resolution of $\sim 0.72 \mu \mathrm{m}$.

\section{Results}

\subsection{Optimisation of $M D+N D$ seeding}

Selected results from the seeding optimisation process are given in Figure 2. As expected, the areal density of the preliminary MD layer was found to increase with increase in both $m$ and $x_{\text {MD }}$ (Figure 2(a)-(c)). However, the large size of the MD particles meant there was an upper limit to the mass of MD powder $\left(m_{\max }=10 \mathrm{mg}\right.$ ) that could be suspended stably in the volume of methanol used. With 1 spray dose $\left(x_{\mathrm{MD}}=1\right)$ a monolayer of MD had still not been formed even using $m_{\max }$ (Figure $\left.2(\mathrm{c})\right)$ and so a second spray dose was required $\left(x_{\mathrm{MD}}=2\right)$ to form a close-packed near-monolayer. Further doses $\left(x_{\mathrm{MD}}>2\right)$ were not beneficial because the MD seeds then formed a partial second layer on top of the first layer of MD seeds, leading to a three-dimensional porous seed layer and a subsequent rough film filled with voids.

Optimisation of the subsequent ND spray dosage showed that if $n$ or $x_{\mathrm{ND}}$ were too low, the ND particles did not cover all exposed $\mathrm{Si}$ areas between the existing MD seeds, resulting in patchy diamond growth and a non-continuous film. For $n \sim 50$ drops, a single ND dose $\left(x_{\mathrm{ND}}=1\right)$ was adequate to achieve a continuous diamond film for ND-only seeding, but for MD+ND seeding a second ND dose $\left(x_{\mathrm{ND}}=2\right)$ was required to ensure all exposed $\mathrm{Si}$ areas were sufficiently seeded to grow an optimal continuous diamond film (Figure 2(e)). For values of $n>50$ drops and $x_{\mathrm{ND}}>2$, the ND particles also coated the surface of the MD seeds, resulting in an exceptionally rough film (Figure $2(d))$. 
For all subsequent experiments on $\mathrm{GaN} / \mathrm{Si}$ and $\mathrm{AlN} / \mathrm{Si}$ substrates, the optimal seeding conditions used were: MD-seeding-only: $m=10 \mathrm{mg}, x_{\mathrm{MD}}=2$; ND-seeding-only: $n=50$ drops, $x_{\mathrm{ND}}=1$; MD+ND seeding: $m=10 \mathrm{mg}, x_{\mathrm{MD}}=2, n=50$ drops, $x_{\mathrm{ND}}=2$.

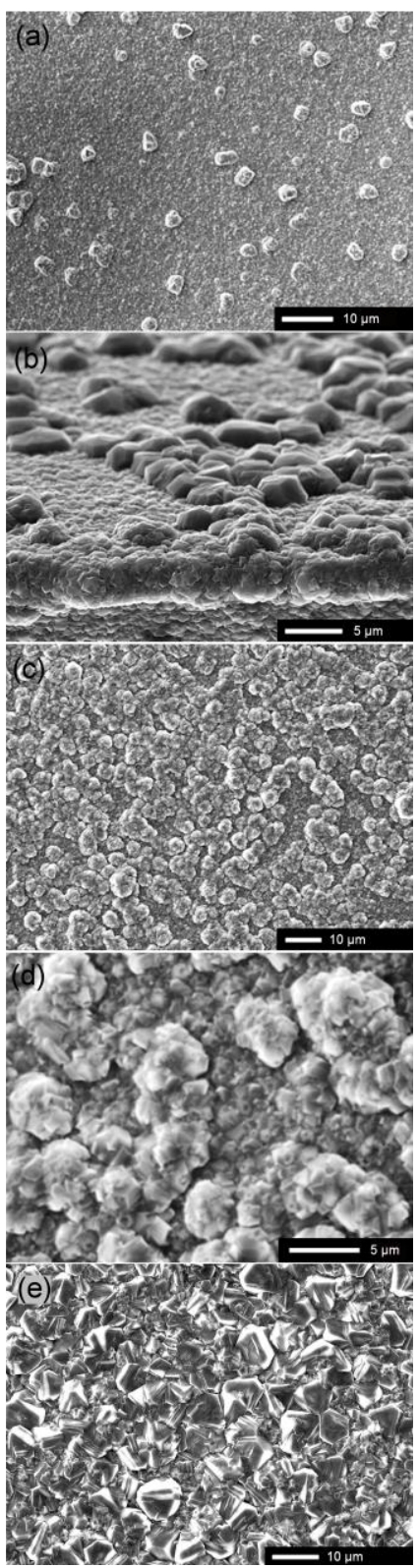

Figure 2. SEM images of selected 3-4 $\mu$ m-thick diamond films grown following different seeding preparations. (a) MD seeds are too sparse $\left(m=5 \mathrm{mg}, \quad x_{\mathrm{MD}}=1, \quad n=50, \quad x_{\mathrm{ND}}=2\right)$; (b) Approximately half a monolayer of MD seeds $(m=7 \mathrm{mg}$, $\left.x_{\mathrm{MD}}=1, \quad n=50, \quad x_{\mathrm{ND}}=2\right) ; \quad(\mathrm{c})$ Approximately $80 \%$ of $\quad$ a monolayer of MD seeds $\left(m=10 \mathrm{~g}, x_{\mathrm{MD}}=1, n=50, x_{\mathrm{ND}}=2\right)$; (d) Approximately $80 \%$ of a monolayer of MD seeds but too many ND doses allows the ND to deposit onto the surface of the MD seeds, leading to a very rough film $\left(m=10 \mathrm{mg}, x_{\mathrm{MD}}=1\right.$, $n=50, x_{\mathrm{ND}}=5$ ); (e) Optimal seeding leads to a near-monolayer of MD seeds and voids filled with ND, resulting in a high quality faceted diamond film ( $\left.m=10 \mathrm{mg}, x_{\mathrm{MD}}=2, n=50, x_{\mathrm{ND}}=2\right)$.

\subsection{Diamond on GaN}

Several thick $(100 \mu \mathrm{m})$ diamond films were grown directly onto GaN/Si substrates at various substrate temperatures and using the optimised ND, MD or MD+ND seeding methods. These thick diamond films are representative of those required to act as efficient heat spreaders in realistic devices. Unfortunately, most of these samples delaminated upon cooling due to poor adhesion and thermal expansion mismatch, as expected [24]. SEM analysis of a nondelaminated sample is shown in Figure 3(a-c), along with XRT images in Figure 3(d) and (e) 
and Raman spectra in Fig.S1 in the Supplementary Information. The SEM images show that although a high-quality diamond film was deposited, the interface between the diamond and the GaN was poor, with evidence of a gap or crack running for many 10 s of $\mu \mathrm{m}$ along the interface. The XRT image in Figure 3(d) also showed an unusual feature at this position, which suggests that this gap was present after growth and cool-down, and was not a result of sectioning for SEM analysis. The 3D cut-away XRT scan in Figure 3(e) shows that the GaN/diamond interface is patchy, with regions where there is good contact between the two layers as well as regions where there is little or no contact. This is not conclusive, however, because of the limited resolution of the XRT system.

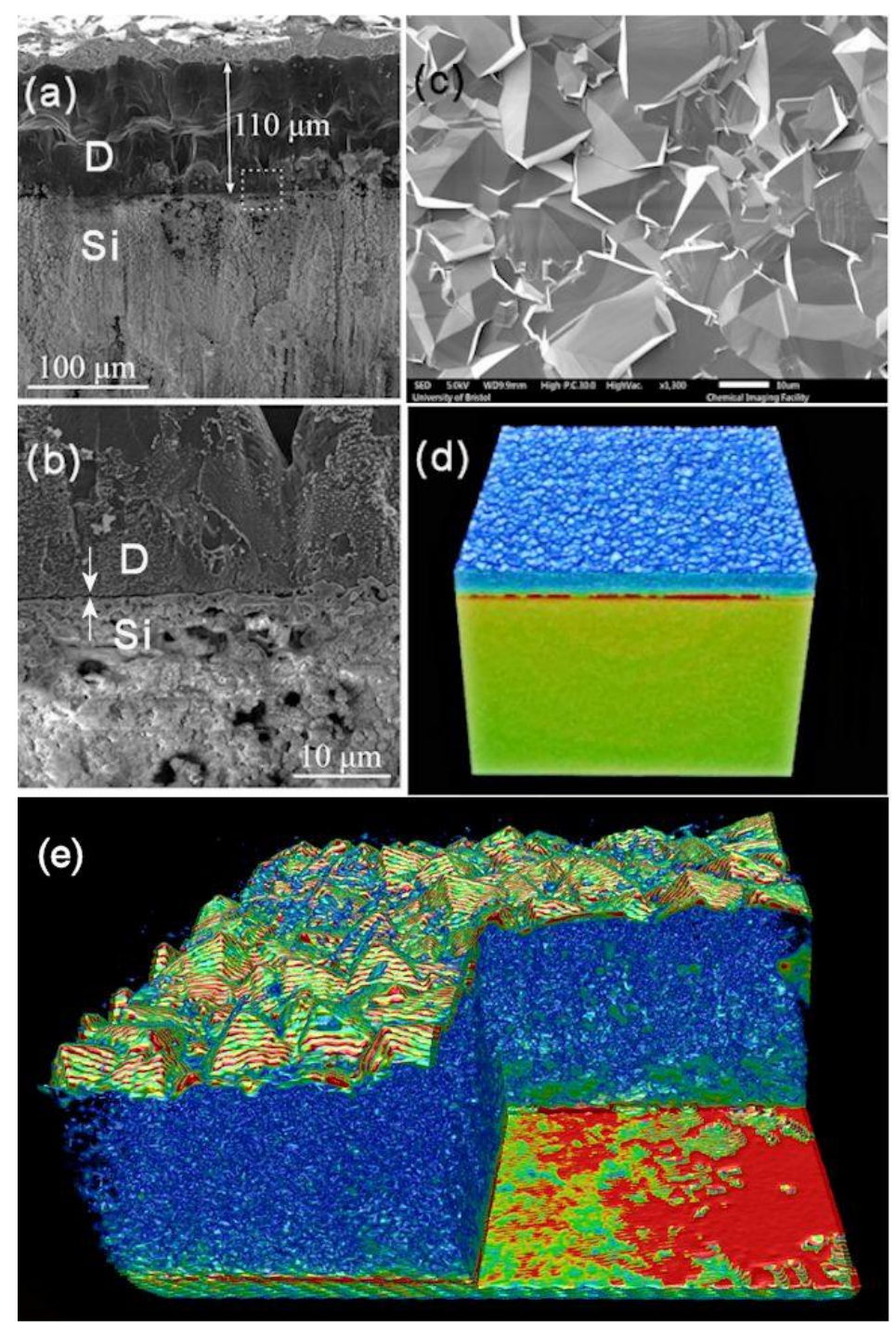

Figure 3. (a) SEM cross-sectional images of a $110 \mu \mathrm{m}$-thick diamond film grown on a GaNon-Si wafer at $750^{\circ} \mathrm{C}$ and (b) close-up of the interface between diamond (D) and $\mathrm{GaN}$ on a Si (Si) substrate, showing a prominent gap (indicated by the arrows) between the diamond and GaN. The GaN layer is too thin to see on this scale (c) Top view of the diamond film 
showing the large crystallite sizes present at the surface and high quality of the facets.

(d) False-colour XRT image of a film grown under similar conditions. The X-rays distinguish regions of different density. We have coloured the diamond blue, and the poor-quality diamond nucleation region lighter blue. Si is green, and we have interpreted the red region as being related to the gap between the diamond and GaN layers. (e) Three-dimensional XRT image of the film with a quadrant of the diamond removed (in software) exposing details of the diamond/GaN interface. The patchy surface highlights the poor interface integrity, with green areas being $\mathrm{GaN}$ that were in contact with the diamond while red show voids or regions with no direct interfacial contact.

TTR was used in an attempt to determine the $\mathrm{TBR}_{\text {eff }}$ of these thick samples, but the high surface roughness resulted in diffuse scattering of the reflected probe beam, preventing accurate measurements. Therefore, three thinner $(2 \mu \mathrm{m})$ and thus, smoother, diamond-on-GaN/Si samples were deposited with MD+ND seeding under identical conditions $\left(750^{\circ} \mathrm{C}, 4 \mathrm{~h}\right)$ allowing successful TTR analysis. Three measurements were made in different positions on each sample, and the mean TBR eff value from all samples was $217 \pm 66 \mathrm{~m}^{2} \mathrm{~K} \mathrm{GW}^{-1}$. This value

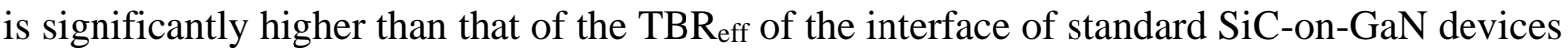
( $330 \mathrm{~m}^{2} \mathrm{~K} \mathrm{GW}^{-1}$ ), showing that diamond grown directly onto $\mathrm{GaN}$ has an inferior thermal interface. This is not surprising, given the images of the poorly adhered, cracked interface seen in Figure 3, and is consistent with our earlier results of attempting to grow diamond directly onto GaN [21].

\subsection{Diamond on AlN}

Diamond $(1-2 \mu \mathrm{m})$ was grown at 750,850 and $950^{\circ} \mathrm{C}$ on $\mathrm{AlN}$-on-Si substrates which had been seeded with MD, ND and MD+ND. However, for MD-only seeding, the adhesion of the diamond to the AlN was so poor that the films readily delaminated, either immediately upon cooling after growth or sometime after. This was presumably due to the very high void ratio at the interface, which we can estimate as $>50 \%$ for the first layer based on the shape of the diamond grains. Indeed, no MD-only-seeded films survived long enough to be TBR tested. For the ND and MD+ND-seeded diamond films which did not delaminate, the film thicknesses were determined by SEM of the cross-section of one representative sample after TTR had been performed. The presented thickness values were measured in various places along the crosssection. TTR data were then fitted to generate values for TBR eff, presented in Table 1. Figure 
4(a) shows a cross-sectional SEM image of one of these films, showing that adherence is now greatly improved with no evidence of cracks or gaps at the interface. The XRT image (Figure 4(b)) also shows no evidence of a crack at the interface. A Raman spectrum of the film is shown as Fig.S2 in the Supplementary Information, along with a lower magnification XRT image (Fig.S3) showing there are no cracks/voids present at the interface over length scales of $\sim 100 \mu \mathrm{m}$. These analyses were representative of many taken with both ND and MD+ND seeding on AlN, suggesting that the interface is now strong, conformal and adherent.

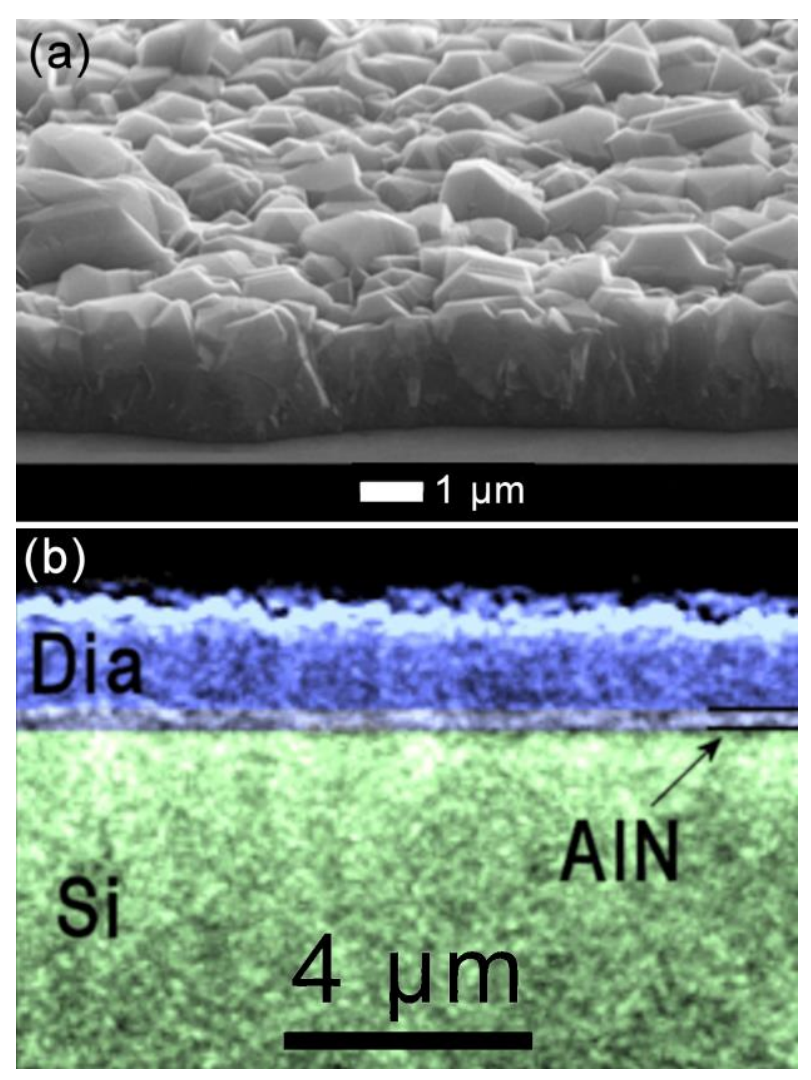

Figure 4. (a) SEM cross-section of a thin diamond film grown on AIN (on $\mathrm{Si}$ ) following MD+ND seeding. The interface is relatively smooth and there are no apparent cracks or voids. (b) XRT image of a cross-section through a similar film. The thin AlN layer can be seen beneath the diamond layer, but this time there are no detectable cracks or gaps at the interface. 
Table 1. Diamond CVD on AlN-on-Si, along with thicknesses and $\mathrm{TBR}_{\mathrm{eff}}$ values measured by TTR. Samples seeded with MD+ND diamond have a significantly lower TBR eff $_{\text {than }}$ those seeded with ND.

\begin{tabular}{|c|c|c|c|c|}
\hline $\begin{array}{c}\text { Growth } \\
\text { Temperature } /{ }^{\circ} \mathrm{C}\end{array}$ & Seeding & Duration $/ \mathrm{h}$ & $\begin{array}{c}\text { Thickness } / \mu \mathrm{m} \\
( \pm 0.1 \mu \mathrm{m})\end{array}$ & $\begin{array}{c}\mathrm{TBR}_{\mathrm{eff}} / \\
\left(\mathrm{m}^{2} \mathrm{~K} \mathrm{GW}^{-1}\right)\end{array}$ \\
\hline 750 & ND & 2.0 & (delaminated) & - \\
\hline 750 & MD+ND & 2.0 & 0.8 & $5.5 \pm 2.6$ \\
\hline 850 & ND & 1.0 & 0.6 & $67 \pm 58$ \\
\hline 850 & MD+ND & 1.0 & 1.2 & $1.47 \pm 0.35$ \\
\hline 950 & ND & 1.0 & 2.3 & $109 \pm 54$ \\
\hline 950 & MD+ND & 1.0 & 2.0 & $3.36 \pm 0.94$ \\
\hline
\end{tabular}

As shown in Table 1, it is clear that the low $\mathrm{TBR}_{\text {eff }}$ values for all samples show that thermal transport is much more effective across the AlN/diamond interface compared to the GaN/diamond interface mentioned previously. Our TBR eff values for ND seeding alone are slightly larger than those reported previously $\left(16 \mathrm{~m}^{2} \mathrm{~K} \mathrm{GW}^{-1}\right.$ [24]) for similar diamond films grown on AlN, probably due to differences in the seeding methods. But comparing the two seeding methods, it is evident that use of MD+ND seeding improved the $\mathrm{TBR}_{\text {eff }}$ for our films by a factor of $30 \times$ or more for the higher temperature growths compared to the equivalent sample seeded by ND alone. Indeed, values for $\mathrm{TBR}_{\mathrm{eff}}<6 \mathrm{~m}^{2} \mathrm{~K} \mathrm{GW}^{-1}$ are quite remarkable and show great promise for device cooling. At the lowest growth temperature $\left(750^{\circ} \mathrm{C}\right)$, the delamination of the ND-seeded sample but not the MD+ND seeded sample, may indicate that the interface is also more adherent in the MD+ND seeding case. These results suggest that the initial layer of MD seeds in the MD+ND seeding provides a much more thermally conductive interface between the layers, allowing a higher quality of diamond and larger grain size at the start of the growth, as well as increasing the overall adherence of the layer.

\section{Discussion and Conclusions}

Diamond CVD growth directly on GaN yielded no significant success, as the diamond had weak bonding to the GaN surface, likely resulting from the lack of viable covalent $\mathrm{Ga}-\mathrm{C}$ bonds. 
This meant that the only adhesive forces between the diamond and GaN layers were likely weak van der Waals interactions. It has recently been shown [33] that strong covalent bonds are essential for efficient phonon transport across an interface between dissimilar materials. Conversely, weak van der Waals bonds lead to phonon reflection at the interface and poor thermal conductivity. In the case of GaN-on-diamond, the problem of the weak interface is compounded with the issue of thermal expansion mismatch between the two layers. SEM and XRT analysis showed that, upon cooling, cracks or small gaps open up at the diamond/GaN interface, resulting in partial or full delamination and negligible thermal conduction through the interface. This suggests that, unfortunately, direct diamond-on-GaN devices are mechanically and thermally unviable, and that an interface layer is essential.

Conversely, the results show that thin $(<200 \mathrm{~nm})$ AlN is a viable interface layer, being chemically and mechanically compatible with $\mathrm{GaN}$, diamond and $\mathrm{Si}$. Consistent with previous reports [24], we find that diamond grown on AlN does not readily delaminate, and exhibits a

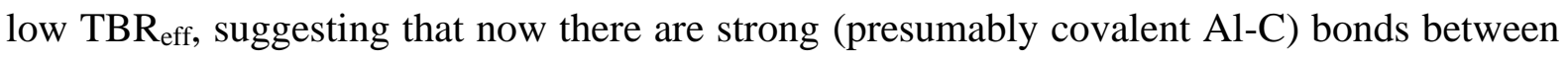
the two layers leading to efficient heat transfer.

Depositing MD+ND in a two-step process to yield a mixed-size diamond seeding prior to CVD growth significantly reduced the thermal resistance at the interface of diamond-on-AlN/Si. Remarkably low $\mathrm{TBR}_{\text {eff }}$ values of $<6 \mathrm{~m}^{2} \mathrm{~K} \mathrm{GW}^{-1}$ were achieved, which can be explained by the MD providing a high surface area at the interface with few grain boundaries. This is aided by the ND component filling in any voids present at the interface, while protecting the exposed AlN surface regions thereby minimising etching and damage [28]. The measured TBR eff $_{\text {of }}$ the mixed seeding samples compare very favourably to the state-of-the art values for amorphous $\mathrm{SiN}_{x}$ and crystalline AIN interlayers reported previously (Table 2). Whilst no details are specified for the seeding method of some of these literature values, it is likely these samples all used nanoseeding because this is the common practice. From Table 2 it is clear that this new 2-step process creates a low-thermal-resistance interface while avoiding potential degradation of the AlN layer.

This result is promising in enabling the fabrication of higher power GaN HEMTs which can be more effectively cooled via a diamond heat spreader than current devices that use GaN-on-SiC. Future studies will be aimed towards optimising the diamond growth itself and investigating the effect of additional layers within the 'substrate stack', without having to add thermally poor conductors as interlayers. 
Table 2. Details of state-of-the-art $\mathrm{TBR}_{\mathrm{eff}}$ values for GaN-on-diamond interlayers. The measurement techniques were either TTR or time-domain thermoreflectance (TDTR) at the ns, ps or fs timescales.

\begin{tabular}{|c|c|c|c|c|c|}
\hline $\begin{array}{c}\text { Interlayer } \\
\text { Material }\end{array}$ & $\begin{array}{c}\text { Interlayer } \\
\text { Thickness / nm }\end{array}$ & Seeding & $\begin{array}{c}\text { TBR }_{\text {eff }} / \\
\left(\mathbf{m}^{2} \mathbf{K ~ G W}^{-1}\right)\end{array}$ & $\begin{array}{c}\text { Measurement } \\
\text { Technique }\end{array}$ & Ref \\
\hline $\begin{array}{c}\text { AlN } \\
\text { Crystalline }\end{array}$ & 130 & ND & $67 \pm 58$ & ns TTR & This work \\
\hline $\begin{array}{c}\text { AlN } \\
\text { Crystalline }\end{array}$ & 130 & Mixed & $1.47 \pm 0.35$ & ns TTR & This work \\
\hline $\begin{array}{c}\text { AlN } \\
\text { Crystalline }\end{array}$ & 250 & ND & $16+8.5 /-6$ & ns TTR & $\begin{array}{c}\text { [Error! } \\
\text { Bookmark } \\
\text { not } \\
\text { defined.] }\end{array}$ \\
\hline $\operatorname{SiN}_{x}$ & 22 & No details & $17.4 \pm 3.0$ & ps TDTR & [34] \\
\hline $\operatorname{SiN}_{x}$ & 31 & No details & $31.8 \pm 5.3$ & ps TDTR & [34] \\
\hline $\operatorname{SiN}_{x}$ & 5 & No details & $9.5+3.8 /-1.7$ & fs TDTR & [35] \\
\hline
\end{tabular}

\section{Acknowledgements}

This work was in part supported by the Engineering and Physical Sciences Research Council (EPSRC) Programme Grant GaN-DaME under EP/P00945X/1. D.J. Wallis would like to acknowledge support of an EPSRC fellowship EP/N01202X/2. The raw data for this work can be found on the Bristol University data depository with doi: \{to be added at proofs stage . 


\section{References}

[1] S. J. Pearton, F. Ren, A. Z. Zhang, K. P. Lee, Fabrication and Performance of GaN Electronic Devices, Mater. Sci. Eng., R30, (2000) 55-212.

[2] U. K. Mishra, P. Parikh, Y.-F. Wu, AlGaN/GaN HEMTs-An Overview of Device Operation and Applications, Proc. IEEE 90, (2002) 1022-1031.

[3] U.K. Mishra, L. Shen, T.E. Kazior, Y.-F. Wu, GaN-Based RF Power Devices and Amplifiers, Proc. IEEE, 96, (2008) 287-305.

[4] A. Bar-Cohen, J. J. Maurer, D. H. Altman, Embedded Cooling for Wide Bandgap Power Amplifiers: A Review. J. Electron. Packag. 141, (2019) 040803.

[5] J.W. Pomeroy, M.J. Urena, B . Lambert, M. Kuball, Operating Channel Temperature in GaN HEMTs: DC Versus RF Accelerated Life Testing. Microelec. Reliability, 55, (2015) 2505-2510.

[6] R.S. Pengelly, S.M. Wood, J.W. Milligan, S.T. Sheppard, W.L. Pribble, A Review of GaN on SiC High Electron-Mobility Power Transistors and MMICs, IEEE Trans. Microwave Theory Tech., 60, (2012) 1764-1783.

[7] H. R. Sun, R. B. Simon, J. W. Pomeroy, D. Francis, F. Faili, D. J. Twitchen, M. Kuball, Reducing GaN-on-Diamond Interfacial Thermal Resistance for High Power Transistor Applications, Appl. Phys. Lett. 106, (2015) 111906.

[8] A. Manoi, J. W. Pomeroy, N. Killat and M. Kuball, Benchmarking of Thermal Boundary Resistance in AlGaN/GaN HEMTs on SiC Substrates: Implications of the Nucleation Layer Microstructure, IEEE Electron Dev. Lett., 31, (2010) 1395-1397.

[9] D. Altman, M. Tyhach, J. McClymonds, S. Kim, S. Graham, J. W. Cho, K. Goodson, D. Francis, F. Faili, F. Ejeckam, S. Bernstein, Analysis and Characterization of Thermal Transport in GaN HEMTs on Diamond Substrates, 14th Intersociety Conf. Thermal and Thermomech. Phenom. in Elect. Sys. (ITherm), Orlando, FL, (2014) 1199-1205.

[10] J. W. Pomeroy, M. Bernardoni, D. C. Dumka, D. M. Fanning, M. Kuball, Low thermal resistance GaN-on-diamond transistors characterized by three-dimensional Raman thermography mapping, Appl. Phys. Lett. 104, (2014) 083513.

[11] P.R. Hageman, J.J. Schermer, P.K. Larsen, GaN Growth on Single-Crystal Diamond Substrates by Metalorganic Chemical Vapour Deposition and Hydride Vapour Deposition, Thin Solid Films 443, (2003) 9-13. 
[12] Y. Taniyasu, M. Kasu, MOVPE growth of single-crystal hexagonal AlN on cubic diamond, J. Cryst. Growth 311, (2009) 2825-2830.

[13] K. Hirama, Y. Taniyasu, and M. Kasu, Heterostructure growth of a single-crystal hexagonal AlN (0001) layer on cubic diamond (111) surface J. Appl. Phys. 108, (2010) 013528.

[14] K. Hirama, Y. Taniyasu, M. Kasu, AlGaN/GaN high-electron mobility transistors with low thermal resistance grown on single-crystal diamond (111) substrates by metalorganic vapor-phase epitaxy, Appl. Phys. Lett. 98, (2011) 162112.

[15] K. Hirama, M. Kasu, Y. Taniyasu, Growth and Device Properties of AlGaN/GaN HighElectron Mobility Transistors on a Diamond Substrate, Jpn. J. Appl. Phys. 51, (2012) 01AG09.

[16] K. Hirama, M. Kasu, Y. Taniyasu, RF High-Power Operation of AlGaN/GaN HEMTs Epitaxially Grown on Diamond, IEEE Electron Device Lett. 33, (2012) 513-515.

[17] K. Hirama, Y. Taniyasu, M. Kasu, Epitaxial Growth of AlGaN/GaN High-Electron Mobility Transistor Structure on Diamond (111) Surface, Jpn. J. Appl. Phys. 51, (2012) 090114.

[18] M. Alomari, A. Dussaigne, D. Martin, N. Grandjean, C. Gaquiere, E. Kohn, AlGaN/GaN HEMT on (111) single crystalline diamond, Electron. Lett. 46, (2010) 299-301.

[19] A. Dussaigne, M. Gonschorek, M. Malinverni, M. A. Py, D. Martin, A. Mouti1, P. Stadelmann, N Grandjean, High-Mobility AlGaN/GaN Two-Dimensional Electron Gas Heterostructure Grown on (111) Single Crystal Diamond Substrate, Jpn. J. Appl. Phys. 49, (2010) 061001.

[20] Q. Wu, Y. Xu, J. Zhou, Y. Kong, T. Chen, Y. Wang, F. Lin, Y. Fu, Y. Jia, X. Zhao, B. Yan, R. Xu, Performance Comparison of GaN HEMTs on Diamond and SiC Substrates Based on Surface Potential Model, ECS J. Solid State Sci. Technol., 6, (2017) 171-178.

[21] P.W. May, H.Y. Tsai, W.N. Wang, J.A. Smith, Deposition of CVD Diamond onto GaN, Diamond Relat. Mater. 15, (2006) 526-530.

[22] B. Hfijek, V. Kohout, V. Flcmr V. Note on Thermodynamic Instability of $\mathrm{M}_{4} \mathrm{C}_{3}$-type Carbides of Gallium Group Metals. Monatshefte fiir Chemic. 1164, (1986) 1157-1164.

[23] M. Oba, T. Sugino, Oriented Growth of Diamond on (0001) Surface of Hexagonal GaN. Diamond Relat. Mater. 10, (2001) 1343-1346.

[24] S. Mandel, C. Yuan, F. Massabuaa, J.W. Pomeroy, J. Cuenca, H. Bland, et al., Thick, Adherent Diamond Films on AlN with Low Thermal Barrier Resistance, ACS Appl. Mater. Interfaces, 11, (2019) 40826-40834. 
[25] A. AlShaikhia, G.P. Srivastava, Thermal conductivity of single crystal and ceramic AlN, J. Appl. Phys., (2008) 103, 083554.

[26] M. D Smith, et al., GaN-on-diamond technology platform: Bonding-free membrane manufacturing process. AIP Advances, 10, (2020) 035306.

[27] P.W. May, Diamond Thin Films: A 21st Century Material, Phil. Trans. R. Soc. Lond. A, 358, (2000) 473-495.

[28] D Liu, D. Francis, F. Faili, C. Middleton, J. Anaya, J.W. Pomeroy, D. J. Twitchen, M. Kuball, Impact of Diamond Seeding on the Microstructural Properties and Thermal Stability of GaN-on-Diamond Wafers for High-Power Electronic Devices, Scripta Materiala 128, (2017) 57-60.

[29] O.J.L. Fox, J.O.P. Holloway, G.M. Fuge, P.W. May, M.N.R. Ashfold, Electrospray Deposition of Diamond Nanoparticle Nucleation Layers for Subsequent CVD Diamond Growth, in Diamond Electronics and Bioelectronics - Fundamentals to Applications III, eds. P. Bergonzo, J.E. Butler, R.B. Jackman, K.P. Loh, M. Nesladek (Mater. Res. Soc. Symp. Proc. 1203, Warrendale, PA, 2010), paper J17-27.

[30] NanoAmando diamond, NanoCarbon Research Institute Co., Japan.

[31] J. W. Pomeroy, R. B. Simon, H. Sun, D. Francis, F. Faili, D. J. Twitchen, and M. Kuball, Contactless Thermal Boundary Resistance Measurement of GaN-on-Diamond Wafers. IEEE Elect. Device Lett. 35, (2014) 1007-1009.

[32] Y. Zhou, J. Anaya, J. Pomeroy, H. Sun, X. Gu, A. Xie, et al., Barrier-Layer Optimization for Enhanced GaN-on-Diamond Device Cooling, ACS Appl. Mater. Interfaces 9, (2017) 34416-34422.

[33] W. M. Waller, J. W. Pomeroy, D. Field, E.J.W. Smith, P.W. May, M. Kuball, Thermal Boundary Resistance of Direct van der Waals Bonded GaN-on-Diamond, submitted to $J$. Appl. Phys. (2020).

[34] J. Cho, D. Francis, D. H. Altman, M. Asheghi, and K. E. Goodson, Phonon conduction in GaN-diamond composite substrates, J. Appl. Phys., 121, (2017) 055105.

[35] L. Yates, et al., Low Thermal Boundary Resistance Interfaces for GaN-on-Diamond Devices, ACS Appl. Mater. Interfaces, 10, (2018) 24302-24309. 